\title{
Cell type-specific gene expression profiling in brain tissue: comparison between TRAP, LCM and RNA-seq
}

\author{
TaeHyun Kim, Chae-Seok Lim E Bong-Kiun Kaang * \\ Department of Biological Sciences, Seoul National University, Seoul 151-747, Korea
}

\begin{abstract}
The brain is an organ that consists of various cell types. As our knowledge of the structure and function of the brain progresses, cell type-specific research is gaining importance. Together with advances in sequencing technology and bioinformatics, cell type-specific transcriptome studies are providing important insights into brain cell function. In this review, we discuss 3 different cell type-specific transcriptome analyses i.e., Laser Capture Microdissection (LCM), Translating Ribosome Affinity Purification (TRAP)/RiboTag, and single cell RNA-Seq, that are widely used in the field of neuroscience. [BMB Reports 2015; 48(7): 388-394]
\end{abstract}

\section{INTRODUCTION}

The brain is composed of various cell types, including neurons and glial cells. Each cell type has a distinct role in making synaptic connections with other cells and controlling their firing patterns. For instance, in rodent and human hippocampi, there are glutamatergic excitatory neurons and $\gamma$-aminobutyric acid (GABA) releasing inhibitory neurons that cause depolarization and hyperpolarization of their postsynaptic neurons, respectively. There are several different ways to classify cell types in the brain, based on cell shape, firing properties (e.g., frequency, rhythmic activity, and pattern of activity), neurotransmitters release, or by specific genes expression (e.g., ligand receptors, ion channels, or cell signaling molecules). Although quite different in their properties, these different classes of neurons must work together efficiently to maintain proper brain function. The importance of each cell type to the overall health of the organism is illustrated by the example of Parkinson's disease, which is known to result from the loss of dopaminergic neurons. In this case, it is clear that the failure of one class of

*Corresponding author. Tel: +82-2-880-9024; Fax: +82-2-884-9577; E-mail: kaang@snu.ac.kr

http://dx.doi.org/10.5483/BMBRep.2015.48.7.218

Received 22 October 2014, Revised 16 November 2014, Accepted 20 January 2015

Keywords: Brain, Cell type-specific, Gene expression profiling, Neuron, Transcriptome neurons can have a drastic effect on brain function and the health of the organism as a whole (1-3). Furthermore, given the complexity of the interactions between different brain cell types, it is likely advantageous to initially focus scientific investigations on a specific cell type.

To date, gene expression profiling studies have largely been focused on brain regions as opposed to different cell types due to the difficulty in isolating cell type-specific transcripts (4-8). This approach may indicate changes in gene expression of the dominant cell types within a particular brain region, but alterations in minor cell populations may be obscured. Often, these minor cell populations are important regulators of their neural network, as with inhibitory interneurons $(9,10)$. Therefore, changes in gene expression in these minor neuron populations may have a significant effect on overall brain function. Moreover, some disease states are the result of altered gene expression in only a few types of brain cells. Therefore, it would be especially valuable for researchers to gather information from specific cell types within the brain.

This review focuses on 3 techniques used for cell type-specific transcriptome analysis in the field of neuroscience: Laser Capture Microdissection (LCM), Translating Ribosome Affinity Purification TRAP/RiboTag, and single cell RNA sequencing (RNA-seq). These techniques are chosen since the sample acquired through these methods can be further analyzed by sequencing techniques. With the improvement of sequencing technologies, these 3 techniques are expected to have increasing importance in future neuroscience research.

\section{Laser Capture Microdissection (LCM)}

In LCM (Fig. 1A), target cells on a tissue section are captured by using an infrared $(11,12)$ or ultraviolet (UV) laser $(13,14)$. When using an IR laser, a polymer membrane is placed on a tissue sample and the laser is focused on a select area. The laser light melts the membrane, which then covers and attaches to the target cells. Target cells are subsequently pulled from the surrounding cells by lifting the membrane. UV light slices around the target cells and separates it from the surrounding tissue. Since LCM requires a very thin tissue slice i.e., 2-15 $\mu \mathrm{m}$, the sample tissue should be fixed before sectioning. If desired, immunostaining may be used to specifically identify a particular population of cells to aid in cell capture. After capture, target cells can be lysed and RNA extracted for further 
(A)
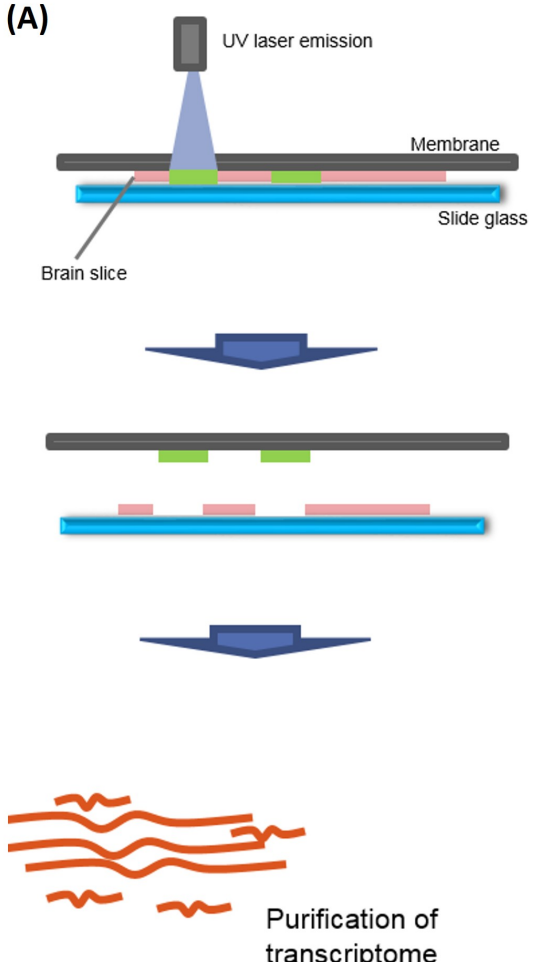

(B)
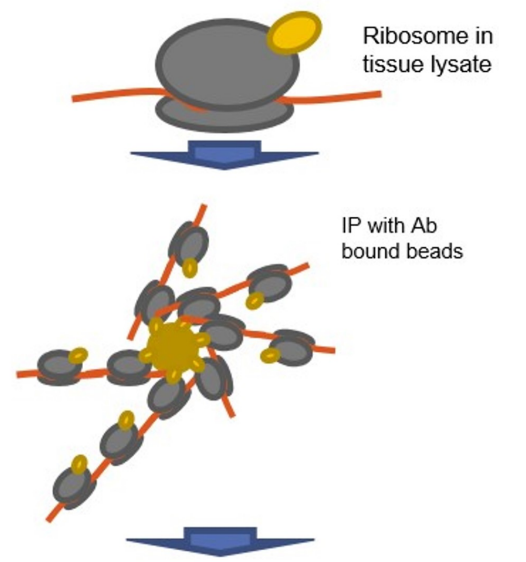

Purification of mRNAs

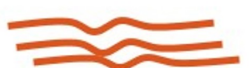

Fig. 1. Sampling transcripts from target cells using LCM or TRAP/ RiboTag (A) Sampling targeted cell population with laser capture microdissection (LCM). A transparent polymer membrane is attached to the brain slice prepared on a slide glass. When the desired cell's border is defined using the software linked to the LCM machine, a UV laser is emitted onto the target region. The UV laser cuts the target cell out from the tissue slice. The target cell population is pulled from the tissue slice and then transcriptome of the specific cell population can be determined. (B) Sampling polysome- bound mRNAs from target cell type with antigen-tagged ribosome. Antigen (HA or GFP)-tagged ribosomal subunit is expressed in the desired cell type using a cell type-specific promoter or Cre recombinase-driven gene expression. After lysing the tissue sample, mRNAs bound to antigen-tagged ribosomes are pulled down using antigen-binding antibodies that are bound to beads. mRNAs are eluted from the beads and then analyzed further. analysis (15).

Although the selection methods of particular cell types are quite different in LCM, we cannot specify the population. When using LCM, however, we can immunostain DCX and MCM2 and simply select cells where the 2 signals colocalize. Another advantage of LCM is that it allows for the collection of specific cell types without the need to use transgenic mice or the expression of external gene constructs such as or RiboTag/ BAC-TRAP and transcriptome in vivo analysis (TIVA). Thus, researchers can save time, money, and exclude the possible effects of exogenous genes influencing the natural gene expression profile.

The major disadvantage of LCM is that the tissue usually goes through a fixation step, which causes non-specific crosslinking of proteins and nucleic acids, and greatly deteriorates the quality of the transcriptome analysis (16). To avoid this problem, chemicals that can reverse the cross-linking $(16,17)$ or freeze-drying instead of chemical fixation (15) have been used. Additionally, when using LCM it is difficult to obtain extrasomatic gene expression profiles due to the complex structure of neurons.

\section{Translating Ribosome Affinity Purification (TRAP) and RiboTag}

TRAP uses antigen-tagged ribosome subunit to immunoprecipitate mRNA bound polysomes in specific cell types. Since in- troduced in $2008(18,19)$, this technique has been used to study the cell type-specific gene expression profiling of any genetically defined cell type from Drosophila to mice and human cells. This method uses bacterial artificial chromosome (BAC) expressing enhanced green fluorescence protein (EGFP)tagged ribosomal protein L10a under a cell type-specific promoter to generate a transgenic mouse line. EGFP-tagged ribosomes, which are present in a particular brain cell population, are then pulled down after brain tissue lysis with an anti-EGFP antibody bound to magnetic beads (Fig. 1B). These isolated ribosomes contain specific mRNA that can be used in subsequent transcriptome analyses (18, 19). Similarly, RiboTag, which is a method introduced in 2009 (20), uses mouse cells that express human influenza hemagglutinin(HA)-tagged Rpl22, which is one of the 79 core proteins that constitute the ribosome particle, under the control of Cre recombinase. As in TRAP, ribosome bound mRNAs are pulled down from brain lysates using an anti-HA antibody bound to magnetic beads and the following steps are basically identical with the TRAP method. Several studies using TRAP indicate that the reproducibility of duplicate samples was $>98 \%$, which is comparable to other techniques such as LCM, Immunopanning and manual dissection (18-24).

The greatest advantage of TRAP and RiboTag is that researchers can obtain mRNAs that are being actively translated at a particular time point (25). Given that there are several 
(A)

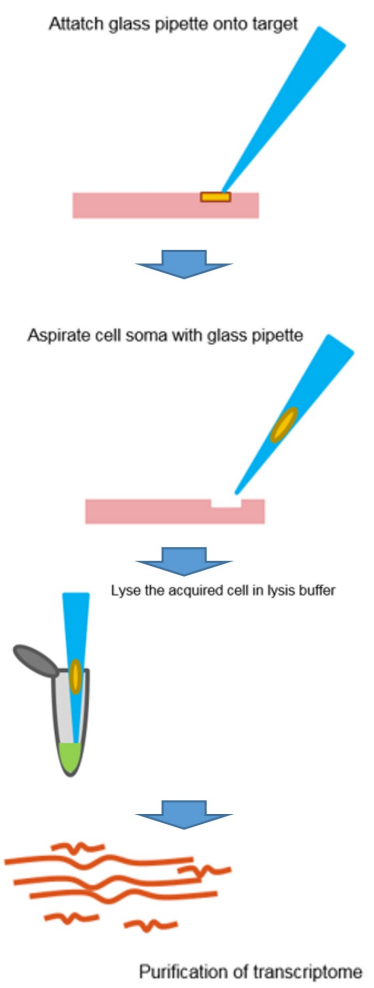

(B)

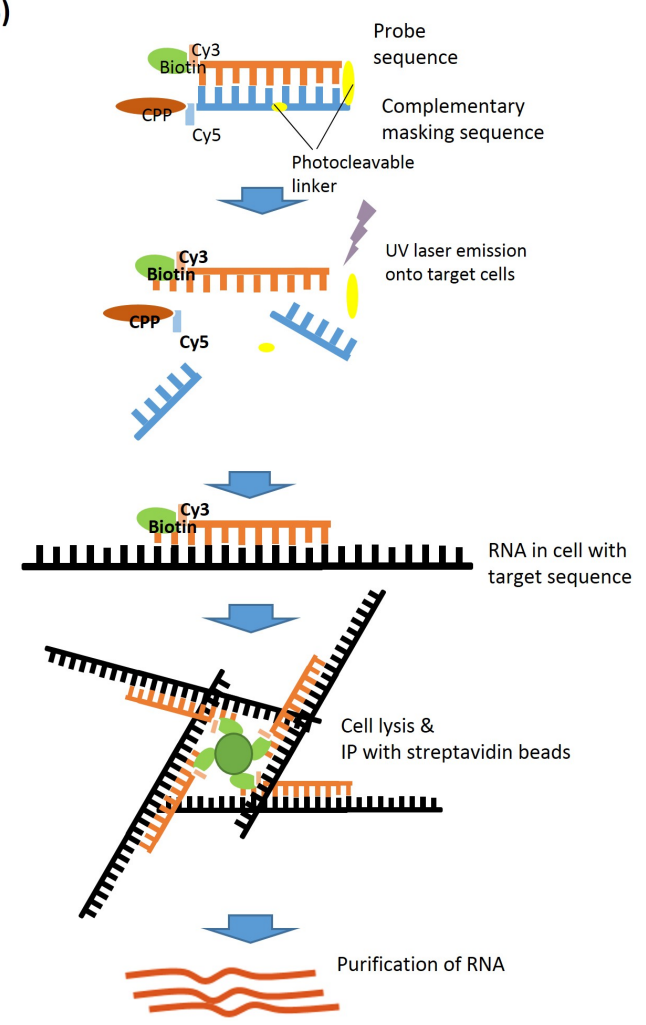

Fig. 2. Single-cell transcriptome analysis. (A) Gathering single cell transcriptome via patch pipette aspiration. A target cell is selected from a tissue slice or neuron culture and patch pipette is attached to the cell membrane. Electrophysiological properties can be measured from the whole-cell preparation. Cell soma components can be acquired by aspiration, and then the transcriptome can be purified from the acquired cell. (B) Capturing target RNAs with activated TIVA-tag. When the tissue is incubated with solution containing TIVA-tag, TIVA-tag can penetrate into the cell by the attached cell penetrating peptide (CPP). UV laser is emitted on desired cell population. The UV laser cleaves the photocleavable linker connecting the masking RNA with the tag, and masking RNA is released from the probe RNA segment, thus the tag is 'activated'. RNAs in the cell containing complementary sequence with the probe is captured by the probe, and the tag is immunoprecipitated with streptavidin beads. Tag-bound RNAs can be eluted and then further analyzed. translational regulation steps following transcription, the use of classical cDNA library microarrays or RNA-seq makes it impossible to discriminate between highly translated mRNAs and other mRNAs. However, using TRAP/RiboTag allows the sample to be enriched with polysome-bound mRNAs rather than monosome-bound mRNAs (19). Additionally, these techniques do not require any fixation or dissociation of target cells from tissue samples. As mentioned above, tissue fixation may cause direct damage to mRNAs or mRNA-ribosome complexes. Furthermore, since TRAP/RiboTag do not require dissociation from the cell soma, the translatome collected with these techniques will contain mRNAs being translated from distal cell structures such as dendritic spines. Given that there is accumulating evidence of synaptic tagging and dendritic protein synthesis $(26,27)$, gathering this information may aid in characterizing synaptic interactions.

The major disadvantage of TRAP/RiboTag is that there must be a specific genetic marker for the cell population of interest. While some cell types can be identified with a specific genetic marker and anatomical localization, many cell types do not have specific gene expression profiles that would allow for isolation. For instance, newborn neurons and neuroblasts can be separated by 2 genetic markers, DCX (newborn neurons and neuroblasts) and MCM2 (neuroblasts). Furthermore, it is hard to choose a subpopulation of specific gene-expressing cells. For instance, calcium/calmodulin-dependent protein kinase II (CaMKII) positive neurons transiently expressing c-fos cannot be distinguished from other basal CaMKII neurons with these techniques. Moreover, a specific issue with TRAP is that the generation of BAC-driven transgenic mice is required, which is quite time-consuming and costly. RiboTag mice utilize a Cre-driven system, and a Cre-driven TRAP mouse model has been generated to solve the problems associated with TRAP (23). These mice contain 'floxed' sequence in their genome so that epitope-tagged ribosomal protein would only be expressed in cells containing Cre-recombinase. RiboTag mouse has a floxed normal C-terminal exon4 of the Rpl22 protein before HA-tagged exon4, thus the HA-tagged exon4 is expressed only after the floxed exon 4 is recombined out by Cre-recombinase. Cre-driven TRAP mouse has a floxed stop cassette upstream the mCherry-tagged Rpl10a protein coding sequence so transcription of the gene would occur when the stop cassette is recombined out by Cre-recombinase.

\section{Single-Cell Transcriptome Analysis}

Recent advances in high-throughput RNA sequencing (RNAseq) technology make it possible to obtain information on single-cell transcriptomes. Patch pipette aspiration is commonly used for single-cell RNA extraction (Fig. 2A) (28-30). This can be performed by using a patch recording setup. Specifically, 
after making a tight seal between the glass patch pipette and the target cell membrane, the cell membrane can be broken by applying negative pressure. At this step, the electrophysiological properties of the cell, such as spontaneous postsynaptic potential or action potential firing properties, can be measured. RNAs from the cell soma can then be pulled into the patch pipette together with other cytosolic components (30).
The advantage of this technique is that the researcher can measure the electrophysiological properties of target cells to confirm their identity. The candidate cell population is further narrowed down by examining the cell's electrophysiological nature to identify desired cell population, which could not be identified with genetic markers such as specific promoter-driven GFP expression alone. However, there are several disadvantages associated with single-cell transcriptome analysis.

Table 1. Some of the recent studies using LCM, TRAP, RiboTag or Single cell transcriptome analysis techniques in neuronal cells

\begin{tabular}{|c|c|c|c|c|}
\hline Method & Author & Published year & Cell types & Findings \\
\hline \multirow[t]{5}{*}{ LCM } & Espina et al. (15) & 2006 & N/A & Introduction LCM protocol \\
\hline & Humerick et al. (37) & 2013 & $\begin{array}{l}\text { Oxytocin or vasopressin magno- } \\
\text { cellular neurons }\end{array}$ & $\begin{array}{l}\text { Transcription factor expression difference } \\
\text { between two cell types }\end{array}$ \\
\hline & Vincent et al. (38) & 2002 & $\begin{array}{l}\text { Hippocampus CA1, CA2, CA3 } \\
\text { neurons }\end{array}$ & $\begin{array}{l}\text { Gene expression profiling in neurons } \\
\text { combining LCM and real-time RT-PCR }\end{array}$ \\
\hline & Herai et al. (39) & 2014 & $\begin{array}{l}\text { Cortical pyramidal neurons in } \\
\text { long-term fixed brain tissue }\end{array}$ & $\begin{array}{l}\text { Showed sRNAs can be detected from } \\
\text { long-term fixed tissues }\end{array}$ \\
\hline & Kohen et al. (40) & 2014 & $\begin{array}{l}\text { Human DG granule cells from } \\
\text { postmortem brain of mental } \\
\text { illness patients }\end{array}$ & $\begin{array}{l}\text { miR-182 shows abnormal expression lev- } \\
\text { el in psychiatric diseases }\end{array}$ \\
\hline \multirow[t]{9}{*}{ TRAP } & Heiman et al. (18) & 2008 & $\begin{array}{l}d r d 1 a, d r d 2, \text { chat, pcp2 positive } \\
\text { neurons }\end{array}$ & Introduction of bacTRAP technology \\
\hline & Doyle et al. (19) & 2008 & $\begin{array}{l}d r d 1 a, d r d 2 \text { positive neurons in } \\
\text { striatum }\end{array}$ & $\begin{array}{l}\text { More number of cell type specific gene } \\
\text { expression, GABAa signaling pathway } \\
\text { gene expression after cocaine admin- } \\
\text { istration in drd1a neurons }\end{array}$ \\
\hline & Thomas et al. (41) & 2012 & GAL4/UAS system & $\begin{array}{l}\text { Application of TRAP technology in } \\
\text { Drosophila sp. }\end{array}$ \\
\hline & Watson et al. (42) & 2012 & $\begin{array}{l}\text { Retinal ganglion cells, rod pho- } \\
\text { toreceptors in Xenopus laevis } \\
\text { retina }\end{array}$ & $\begin{array}{l}\text { Application of TRAP technology in X. } \\
\text { laevis }\end{array}$ \\
\hline & Schmidt et al. (25) & 2012 & $\begin{array}{l}\text { S100a10 gene expressing neu- } \\
\text { rons in mouse brain cortex }\end{array}$ & $\begin{array}{l}\text { Identification of neurons responding to } \\
\text { antidepressant treatment. }\end{array}$ \\
\hline & Dalal et al. (22) & 2013 & $\begin{array}{l}\text { Hypocretin neurons in hypothal- } \\
\text { amus }\end{array}$ & Genes important in sleep regulation \\
\hline & Tryon et al. (43) & 2013 & Ubiquitous or melanocyte & $\begin{array}{l}\text { Application of TRAP technology in } \\
\text { Zebrafish }\end{array}$ \\
\hline & Hupe et al. (23) & 2014 & Cre-dependent & $\begin{array}{l}\text { Development of Cre-dependent TRAP } \\
\text { method }\end{array}$ \\
\hline & Kratz et al. (24) & 2014 & Purkinje neurons & $\begin{array}{l}\text { Detailed gene expression profiling in } \\
\text { purkinje cells }\end{array}$ \\
\hline \multirow[t]{2}{*}{ RiboTag } & Sanz et al. (20) & 2009 & Cre-dependent & Introduction of RiboTag technology \\
\hline & Brackett et al. (44) & 2013 & Eno2 (neuron specific) & Detection of FMR1 transcript isoforms \\
\hline \multirow[t]{5}{*}{$\begin{array}{l}\text { Single-Cell transcriptome } \\
\text { analysis }\end{array}$} & Gustincich et al. (45) & 2004 & Dopaminergic neuron in retina & $\begin{array}{l}\text { Gene expression profiling of DA neurons } \\
\text { in retina }\end{array}$ \\
\hline & Esumi et al. (46) & 2008 & GABAergic neuron progenitors & $\begin{array}{l}\text { Single-cell microarray analysis method } \\
\text { development }\end{array}$ \\
\hline & Qiu et al. (30) & 2012 & Cultured hippocampal neurons & $\begin{array}{l}\text { Introduction of patch pipette aspiration } \\
\text { technique with electrophysiology re- } \\
\text { cording }\end{array}$ \\
\hline & Hickman et al. (47) & 2013 & Microglia & $\begin{array}{l}\text { Identification of genes involved in micro- } \\
\text { glia target sensing }\end{array}$ \\
\hline & Alldred et al. (48) & 2014 & Hippocampal CA1 & $\begin{array}{l}\text { Gene expression profiling in Ts65Dn } \\
\text { mouse (Alzheimer's disease, Down } \\
\text { syndrome model mouse) }\end{array}$ \\
\hline
\end{tabular}


First, extremely small quantities of RNA can be a significant issue during PCR amplification of transcripts and the subsequent construction of a cDNA library. Although progress continues to be made in PCR to reduce errors, this concern remains a major concern for single-cell transcriptome analysis $(31,32)$. Moreover, given that only cell soma contents can be extracted with this technique, RNAs localized in distal cell structures such as dendritic spines are ignored with this method. Furthermore, aspiration places a mechanical stress on the cell being analyzed, which could result in an abnormal transcriptome profile. To overcome this, transcriptome in vivo analysis (TIVA)tag was recently developed (33).

TIVA-tag is a RNA probe that can penetrate the cell membrane and capture RNAs containing designed target sequence (Fig. 2B). When targeting mRNAs in the cell, for example, the probe sequence could be RNA poly-U sequence so that the probe can bind to the poly-A tail present in the mRNAs. TIVA-tag acquires its cell-type specificity by by UV laser activation. Before being activated by UV laser, the probe sequence is masked by a complementary sequence linked to the tag with a photocleavable linker. UV laser cleaves this linker, and the masking sequence gets detached from the probe sequence thus the TIVA-tag is activated. As the probe is biotinylated, TIVA-tags can be immunoprecipitated with streptavidin beads. Captured RNAs can be eluted from these immunoprecipitated tags and then further analyzed. Since the probe-bound mRNAs are pulled down from tissue lysates rather than aspirated from a cell soma, mechanical stress would be avoided. Moreover, this method allows for the collection of mRNA from distal cell structures (33).

\section{CONCLUSION}

Several methods can be used to analyze gene expression in specific cell types in the complex nervous system (Table 1). LCM gives precise sampling from a visualized area; however, fixation, sectioning, and UV irradiation of tissue may cause damage to RNA. Moreover, it is difficult to obtain a sample from very complex neural structures with LCM. TRAP and RiboTag allow relatively easy sampling from both simple and complex neural structures. However, the cell type must be genetically defined, which requires the use of transgenic animals. TRAP and RiboTag only collect polysome-bound mRNAs, which may be beneficial depending on the goal of the study. Single-cell transcriptome analysis allows the collection of mRNA from specific cell types, while also measuring their electrophysiological properties. However, the small amount of RNA may hamper expression analysis.

Recent studies of cell type-specific research are aimed at narrowing the target cell population. This reduces sample population into cells with a more homogeneous gene expression profile, which enables a more sensitive analysis of gene expression. However, when obtaining a gene expression profile from a minor cell population, the limited starting material may distort the transcriptome data. This mainly results from errors in cDNA synthesis or PCR amplification $(31,32)$. To overcome these problems, sequencing methods without sample amplification are being developed (34-36).

Cell type-specific studies can also aid humanity to fight against brain-related diseases. Specifying the cell types that are affected in the disease or identifying cell populations that respond to known therapeutic agents will give us greater understanding of what causes the disease state and how to cure it. By limiting the target of therapeutic agents to specific cell populations, we can design even more powerful drugs with minimal side-effects.

At first glance, cell type-specific gene expression profiles may show a more complex picture of the brain. However, it should be noted that these analyses could result in a more homogenous gene expression profile within a specific population of cells, and be altogether more reliable than tissue-based data. Through these cell-type specific transcriptome analyses, we can not only obtain a greater understanding of brain structure and function, but also allow for the identification of therapeutic targets for brain-related diseases. Akin to making a high-resolution image, a study with a single brain cell type would reveal a very small part of a much bigger picture. However, when the behavior and properties of the various brain cell populations are merged together, we are granted a more precise and clear picture of the brain than could have been drawn using previous approaches.

\section{ACKNOWLEDGEMENTS}

This work was supported by the National Research Foundation of Korea (NRF) grant funded by the Korea government (MSIP) (National Honor Scientist Program). T.K. is supported by POSCO TJ Park foundation.

\section{REFERENCES}

1. Greene JG (2006) Gene expression profiles of brain dopamine neurons and relevance to neuropsychiatric disease. J Physiol 575, 411-416

2. Liu G, Yu J, Ding J et al (2014) Aldehyde dehydrogenase 1 defines and protects a nigrostriatal dopaminergic neuron subpopulation. J Clin Invest 124, 3032-3046

3. Wang HS, Toh J, Ho P, Tio M, Zhao Y and Tan EK (2014) In vivo evidence of pathogenicity of VPS35 mutations in the Drosophila. Mol Brain 7, 73

4. Barco A, Patterson SL, Alarcon JM et al (2005) Gene expression profiling of facilitated L-LTP in VP16-CREB mice reveals that BDNF is critical for the maintenance of LTP and its synaptic capture. Neuron 48, 123-137

5. Hawrylycz MJ, Lein ES, Guillozet-Bongaarts AL et al (2012) An anatomically comprehensive atlas of the adult human brain transcriptome. Nature 489, 391-399

6. Iwazaki T, McGregor IS and Matsumoto I (2008) Protein expression profile in the amygdala of rats with methamphetamine-induced behavioral sensitization. Neurosci 
Lett $435,113-119$

7. Lein ES, Hawrylycz MJ, Ao N et al (2007) Genome-wide atlas of gene expression in the adult mouse brain. Nature 445, 168-176

8. Hagihara H, Ohira K, Takao K and Miyakawa T (2014) Transcriptomic evidence for immaturity of the prefrontal cortex in patients with schizophrenia. Mol Brain 7, 41

9. Verret L, Mann EO, Hang GB et al (2012) Inhibitory interneuron deficit links altered network activity and cognitive dysfunction in Alzheimer model. Cell 149, 708-721

10. Yizhar O, Fenno LE, Prigge M et al (2011) Neocortical excitation/inhibition balance in information processing and social dysfunction. Nature 477, 171-178

11. Bonner RF, Emmert-Buck M, Cole K et al (1997) Laser capture microdissection: molecular analysis of tissue. Science 278, 1481,1483

12. Emmert-Buck MR, Bonner RF, Smith PD et al (1996) Laser capture microdissection. Science 274, 998-1001

13. Schutze K, Becker I, Becker KF et al (1997) Cut out or poke in-the key to the world of single genes: laser micromanipulation as a valuable tool on the look-out for the origin of disease. Genet Anal 14, 1-8

14. Kolble K (2000) The LEICA microdissection system: design and applications. J Mol Med (Berl) 78, B24-25

15. Espina V, Wulfkuhle JD, Calvert VS et al (2006) Laser-capture microdissection. Nat Protoc 1, 586-603

16. Xiang CC, Mezey E, Chen M, Key S, Ma L and Brownstein MJ (2004) Using DSP, a reversible cross-linker, to fix tissue sections for immunostaining, microdissection and expression profiling. Nucleic Acids Res 32, e185

17. Namimatsu S, Ghazizadeh M and Sugisaki Y (2005) Reversing the effects of formalin fixation with citraconic anhydride and heat: a universal antigen retrieval method. J Histochem Cytochem 53, 3-11

18. Heiman M, Schaefer A, Gong S et al (2008) A translational profiling approach for the molecular characterization of CNS cell types. Cell 135, 738-748

19. Doyle JP, Dougherty JD, Heiman M et al (2008) Application of a translational profiling approach for the comparative analysis of CNS cell types. Cell 135, 749-762

20. Sanz E, Yang L, Su T, Morris DR, McKnight GS and Amieux PS (2009) Cell-type-specific isolation of ribosome-associated mRNA from complex tissues. Proc Natl Acad Sci U S A 106, 13939-13944

21. Okaty BW, Sugino K and Nelson SB (2011) A quantitative comparison of cell-type-specific microarray gene expression profiling methods in the mouse brain. PLoS One 6, e16493

22. Dalal J, Roh JH, Maloney SE et al (2013) Translational profiling of hypocretin neurons identifies candidate molecules for sleep regulation. Genes Dev 27, 565-578

23. Hupe M, Li MX, Gertow Gillner K, Adams RH and Stenman JM (2014) Evaluation of TRAP-sequencing technology with a versatile conditional mouse model. Nucleic Acids Res 42, e14

24. Kratz A, Beguin P, Kaneko M et al (2014) Digital expression profiling of the compartmentalized translatome of Purkinje neurons. Genome Res 8, 1396-1410

25. Schmidt EF, Warner-Schmidt JL, Otopalik BG, Pickett SB, Greengard P and Heintz N (2012) Identification of the cortical neurons that mediate antidepressant responses. Cell 149, 1152-1163

26. Martin KC and Kosik KS (2002) Synaptic tagging -- who's it? Nat Rev Neurosci 3, 813-820

27. Ostroff LE, Fiala JC, Allwardt B and Harris KM (2002) Polyribosomes redistribute from dendritic shafts into spines with enlarged synapses during LTP in developing rat hippocampal slices. Neuron 35, 535-545

28. Spaethling JM, Piel D, Dueck H et al (2014) Serotonergic neuron regulation informed by in vivo single-cell transcriptomics. FASEB J 28, 771-780

29. Moroz LL and Kohn AB (2013) Single-neuron transcriptome and methylome sequencing for epigenomic analysis of aging. Methods Mol Biol 1048, 323-352

30. Qiu S, Luo S, Evgrafov O et al (2012) Single-neuron RNA-Seq: technical feasibility and reproducibility. Front Genet 3, 124

31. Saliba AE, Westermann AJ, Gorski SA and Vogel J (2014) Single-cell RNA-seq: advances and future challenges. Nucleic Acids Res 42, 8845-8860

32. Macaulay IC and Voet T (2014) Single cell genomics: advances and future perspectives. PLoS Genet 10, e1004126

33. Lovatt D, Ruble BK, Lee J et al (2014) Transcriptome in vivo analysis (TIVA) of spatially defined single cells in live tissue. Nat Methods 11, 190-196

34. Ozsolak F and Milos PM (2011) Single-molecule direct RNA sequencing without cDNA synthesis. Wiley Interdiscip Rev RNA 2, 565-570

35. Ozsolak F and Milos PM (2011) Transcriptome profiling using single-molecule direct RNA sequencing. Methods Mol Biol 733, 51-61

36. Ozsolak F, Platt AR, Jones DR et al (2009) Direct RNA sequencing. Nature 461, 814-818

37. Humerick M, Hanson J, Rodriguez-Canales J et al (2013) Analysis of transcription factor mRNAs in identified oxytocin and vasopressin magnocellular neurons isolated by laser capture microdissection. PLoS One 8, e69407

38. Vincent VA, DeVoss JJ, Ryan HS and Murphy GM Jr (2002) Analysis of neuronal gene expression with laser capture microdissection. J Neurosci Res 69, 578-586

39. Herai RR, Stefanacci L, Hrvoj-Mihic B et al (2014) Micro RNA detection in long-term fixed tissue of cortical glutamatergic pyramidal neurons after targeted laser-capture neuroanatomical microdissection. J Neurosci Methods 235c, 76-82

40. Kohen R, Dobra A, Tracy JH and Haugen E (2014) Transcriptome profiling of human hippocampus dentate gyrus granule cells in mental illness. Transl Psychiatry 4, e366

41. Thomas A, Lee PJ, Dalton JE et al (2012) A versatile method for cell-specific profiling of translated mRNAs in Drosophila. PLoS One 7, e40276

42. Watson FL, Mills EA, Wang X, Guo C, Chen DF and Marsh-Armstrong N (2012) Cell type-specific translational profiling in the Xenopus laevis retina. Dev Dyn 241, 1960-1972

43. Tryon RC, Pisat N, Johnson SL and Dougherty JD (2013) Development of translating ribosome affinity purification for zebrafish. Genesis 51, 187-192

44. Brackett DM, Qing F, Amieux PS, Sellers DL, Horner PJ 
and Morris DR (2013) FMR1 transcript isoforms: association with polyribosomes; regional and developmental expression in mouse brain. PLoS One 8, e58296

45. Gustincich S, Contini M, Gariboldi M et al (2004) Gene discovery in genetically labeled single dopaminergic neurons of the retina. Proc Natl Acad Sci U S A 101, 5069-5074

46. Esumi S, Wu SX, Yanagawa $Y$, Obata K, Sugimoto $Y$ and Tamamaki N (2008) Method for single-cell microarray analysis and application to gene-expression profiling of
GABAergic neuron progenitors. Neurosci Res 60, 439-451 47. Hickman SE, Kingery ND, Ohsumi TK et al (2013) The microglial sensome revealed by direct RNA sequencing. Nat Neurosci 16, 1896-1905

48. Alldred MJ, Lee SH, Petkova E and Ginsberg SD (2014) Expression profile analysis of hippocampal CA1 pyramidal neurons in aged Ts65Dn mice, a model of Down syndrome (DS) and Alzheimer's disease (AD). Brain Struct Funct [Epub Ahead of Print] 